\title{
VULNERABILIDAD EN ESCUELAS PRIVADAS SUBVENCIONADAS CHILENAS: DESAFÍOS DOCENTES DESDE LA VISIÓN DIRECTIVA
}

\author{
Nibaldo Benavides-Moreno ${ }^{1}$ \\ Sebastián Donoso-DíaZ ${ }^{2}$ \\ Daniel Reyes-Araya ${ }^{3}$
}

\begin{abstract}
RESUMEN: Este artículo revisa los alcances que para el director escolar reportan las condiciones de vulnerabilidad de un estudiante e influencias que tiene el trabajo docente en su desempeño académico. Corresponde a un estudio exploratorio, basado en entrevistas a directivos de escuelas particular-subvencionadas con estudiantes en esta condición. El análisis permite evidenciar el pensamiento directivo y las dinámicas docentes, identificando nudos críticos que de ser atendidos incidirían positivamente en la organización de la enseñanza en este contexto. A partir de los antecedentes se formulan propuestas en el plano de las políticas públicas que tiendan a la orientación y desarrollo del liderazgo y la gestión pedagógica en este marco.
\end{abstract}

Palabras-clave: Vulnerabilidad. Educación particular subvencionada. Desempeño docente. Chile.

\section{VULNERABILITY IN CHILEAN SUBSIDIZED PRIVATE SCHOOLS: TEACHING CHALLENGES FROM THE BOARD OF DIRECTORS}

\begin{abstract}
The article reviews that the student's conditions of vulnerability and the influence of teaching work on their academic performance are reported to the school principal. It corresponds to an exploratory study, based on interviews with executives of private-subsidized schools with students in this condition. The analysis makes it possible to demonstrate the directive thinking, and the teaching dynamics, identifying critical nodes that, if taken care of, would have a positive impact on the organization of teaching in this context. Based on the background, proposals are made at the level of public policies that tend to guide and develop leadership, and pedagogical management in this framework
\end{abstract}

Keywords: Vulnerability. Subsidized private education. Teaching performance. Chile.

Este proyecto ha sido financiado por el Fondo UTALCA-IIDE, 2019-06.

1.Universidad de Talca, Facultad de Ciencias de la Educación, Instituto de Investigación y Desarrollo Educacional - Talca, Chile. E-mail: nbenavides@utalca.cl

2.Universidad de Talca, Facultad de Ciencias de la Educación, Instituto de Investigación y Desarrollo Educacional - Talca, Chile.E-mail: sdonoso@utalca.cl

3.Universidad de Talca, Facultad de Ciencias de la Educación, Instituto de Investigación y Desarrollo Educacional - Talca, Chile.E-mail: dreyes@utalca.cl 


\title{
VULNERABILIDADE NAS ESCOLAS PARTICULARES SUBSIDIADAS CHILENAS: DESAFIOS DE ENSINO DO CONSELHO DE ADMINISTRAÇÃO
}

\begin{abstract}
RESUMO: Este artigo revisa o alcance que condições de vulnerabilidade do aluno e a influência do trabalho docente em seu desempenho acadêmico têm para o diretor da escola. Corresponde a um estudo exploratório, baseado em entrevistas com executivos de escolas subsidiadas por particulares com alunos nessa condição. A análise possibilita demonstrar o pensamento diretivo e a dinâmica do ensino, identificando nós críticos que, se atendidos, teriam um impacto positivo na organização do ensino nesse contexto. Com base nos antecedentes, são realizadas propostas no nível de políticas públicas que tendam a orientar e desenvolver a liderança e a gestão pedagógica nesse âmbito.
\end{abstract}

Palavras-chave: Vulnerabilidade. Educação privada subsidiada. Desempenho docente. Chile.

\section{Introducción}

$\mathrm{E}$

1 concepto vulnerabilidad se asocia a la reducida capacidad que un individuo o grupo social tiene para adaptarse a ciertas circunstancias. Flores (2009) señala que es un atributo de contextos sociales, nunca de personas, por lo que clasificar a un estudiante como vulnerable se refiere directamente a estigmatización y marginación. Beltrán (2014) considera la vulnerabilidad educativa como:

[...] el conjunto de condiciones (materiales y simbólicas, de orden objetivo y subjetivo) que debilitan el vínculo de escolarización de un alumno. La noción de vulnerabilidad educativa exige dar cuenta de las interacciones entre estas dimensiones, con particular atención a aquellos factores propiamente escolares (DIRECCIÓN..., 2009, p. 3).

En este contexto los directivos escolares en Chile asumen un rol clave, pues las condiciones de vulnerabilidad de una familia son traspasadas al sistema escolar y no solo afectan a estudiantes, sino también el desempeño docente. Ello implica que abordar pedagógicamente a jóvenes deprivados socialmente dificulta y hace más compleja su labor (ESPINOZA et al., 2012).

En este plano Weinstein y Muñoz (2012) plantean que las nuevas políticas concuerdan con la tendencia internacional, presionando a los directores de escuelas por medio de políticas de rendición de cuentas. Lo que se potenció con la promulgación de la Ley n. 20.501 ${ }^{1}$ del 2011, que demandó a los directivos actuar más rigurosamente sobre la continuidad laboral y desarrollo profesional de sus profesores, en el entendido que las escuelas en condiciones de vulnerabilidad necesitan más profesores altamente calificados y son las que tienden a presentar un menor número (RIVERO, 2015). Asimismo, al ser escuelas de propiedad y administración privada, los sostenedores exigen a sus directores obtener resultados, siendo los más relevantes la mantención o aumento de la matrícula y el logro de determinados puntajes en pruebas estandarizadas de medición de aprendizajes. Por lo tanto, su rol principal es evaluar el cumplimiento de estos indicadores, lo que condiciona eventualmente la permanencia del director en su función. Adicionalmente, tiene la responsabilidad de rendir cuenta legal y financiera a la Superintendencia de Educación². 
La legislación entrega al sostenedor o empresario privado la potestad de contratar y poner fin al contrato de un docente cada año escolar (marzo-febrero), tarea regularmente delegada al director del establecimiento. Siendo la centralidad del director, una práctica muy arraigada en la gestión del establecimiento, y en referencia directa a la continuidad de sus docentes, por la cual estudiar esta temática desde este actor es clave, en poder determinar su incidencia en las trayectorias profesionales de los docentes y -tambiénestablecer mecanismos de perfeccionamiento ajustado a las variables y criterios que demandan para estas realidades.

Consistente con ello, el objetivo de esta investigación fue analizar las opiniones de directores de establecimientos escolares particular-subvencionadas ${ }^{3}$ respecto del trabajo que desarrollan sus docentes, en función de dos razones relevantes: 1) identificar las principales características que poseen, señalando la influencia que tienen en el desempeño académico de los estudiantes, identificando problemas y desafíos; para luego 2) formular propuestas de apoyo y orientación para potenciar la gestión pedagógica en contextos escolares vulnerables.

\section{Antecedentes Conceptuales}

\section{La Temática de la Vulnerabilidad en Educación en Chile}

El Ministerio de Educación establece como estudiantes vulnerables:

[...] aquellos que presentan ciertas características de orden socioeconómico tales como: pobreza, marginalidad, disfuncionalidad e ingreso familiar, nivel educativo de los padres, entre otros, como factores que gatillan la vulnerabilidad del estudiante, condicionantes que son traspasadas y afectan al sistema escolar (MINEDUC, 2008a, p. 1-2).

El MINEDUC (2008b) define como guía para la calidad de la docencia, el Marco para la Buena Enseñanza, especificando tres competencias esenciales de todo docente: Dominio del contenido disciplinar; Dominio de la didáctica del área que enseñan y; Habilidades socioemocionales para construir un clima de aula que promueva el aprendizaje. No obstante, muchos docentes que trabajan en contextos vulnerables en Chile, emplean regularmente prácticas pedagógicas basadas en la reproducción de contenidos dispuestos en los marcos curriculares que conllevan al desarrollo de conocimientos abstractos y estimulación de la memoria, no considerando el entorno y la realidad de sus estudiantes (ROMÁN; CARDEMIL, 2000; CARDEMIL et al., 2006; ROMÁN, 2003).

Fullan y Hargreaves (1999) expresan que:

[...] los enormes desafíos que conlleva la enseñanza en contextos de diversidad y vulnerabilidad y, junto con ello, la necesidad de hacer más equitativa la distribución social del conocimiento tanto en la sociedad como al interior de la escuela, trae consigo la necesidad de fortalecer las competencias de los profesores que componen las UTP que puedan trabajar en contextos sociales y pedagógicos muy complejos, cuya magnitud excede los preceptos de las metodologías tradicionales de la enseñanza (apud BELTRÁN, 2014, p. 948).

Por otro lado, la conformación del sistema escolar chileno, en palabras de Bellei, no contribuye a facilitar los resultados educativos en sectores vulnerables, ya que: 
[...] la segregación socioeconómica y la segregación académica de la población escolar no son rasgos anecdóticos de los sistemas escolares, sino que constituyen factores relevantes de la (in) equidad educativa, en tanto afectan diferencialmente las oportunidades y logros de aprendizaje de los alumnos (2013, p. 339).

En el país, tres son las agencias estatales encargadas de atender a estudiantes en condición de vulnerabilidad: Ministerio de Educación, Junta Nacional de Auxilio Escolar y Becas, Ministerio de Desarrollo Social (en adelante MINEDUC, JUNAEB ${ }^{4}$ y MDS5). El MINEDUC (2008a) definió la categoría de "alumnos vulnerables" para la población escolar que recibe una asignación financiera especial en el marco de la "Ley de Subvención Escolar Preferencial” (SEP) a partir del año 2008. La JUNAEB mide la vulnerabilidad escolar mediante el "Índice de Vulnerabilidad Estudiantil (IVE)". Hasta el 2006 se calculaba a partir de la información levantada por las encuestas escolares anuales. En el año 2007 se modifica creándose el IVE - SINAE7 que refleja además la condición de riesgo asociada a los/as estudiantes de cada establecimiento, incorporando una multiplicidad de factores del ciclo educacional del estudiante (JUNAEB, 2015).

En relación a la oferta educacional en el país, el sector particular subvencionado atiende al $25 \%$ de los estudiantes vulnerables, evidenciando alta segregación al respecto. Asimismo, casi el 70\% de la matrícula vulnerable básica total se agrupa mayoritariamente en establecimientos que persiguen fines de lucro o ganancia (BRUNNER; ELACQUA, 2006, VILLARROEL, 2014).

Dados los cambios en la legislación, que permite a los establecimientos educacionales privados obtener esta subvención, el sector particular subvencionado atienden más población escolar vulnerable que hace 5 años (VILLARROEL, 2014). Sin embargo, este sector según Elacqua y Pacheco (2006) y GarcíaHuidobro (2007) impacta más negativamente en la segregación social que el sector público (municipal). Mientras la composición social de las escuelas municipales es uniforme -la mayoría de los colegios tienen un número similar de alumnos vulnerables-, en el particular subvencionado los estudiantes vulnerables se encuentran concentrados en algunos establecimientos.

\section{La Práctica Docente en Contextos Vulnerables}

Se considera que el proceso de enseñanza aprendizaje ocurre esencialmente en el aula, y el factor principal de los resultados de los estudiantes es la dinámica de interacción socio-afectiva, cognitiva y pedagógica entre el docente y el estudiante en el aula y en la escuela. Esta relación dual es importante cuando nos referimos a estudiantes que provienen de familias más carentes y vulnerables (GARCÍA-HUIDOBRO, 1999; ARELLANO, 2000; BELLEI, 2001; COX, 2001). Román afirma que:

[...] la práctica pedagógica de los docentes de las escuelas vulnerables, está fuertemente ligada a modelos que ellos construyen y reproducen sobre las potencialidades de sus propios estudiantes, generando y estigmatizando formas estables e inefectivas en el proceso de enseñanza aprendizaje, que se ve reflejado directamente en su práctica de aula (2003, p. 114).

Los docentes coinciden que su principal debilidad está en el manejo de estrategias pedagógicas y disciplinares adecuadas a ambientes específicos que aseguren procesos de aprendizaje efectivos, generando un clima aula acorde, de comunicación, afecto y confianza. En un contexto de vulnerabilidad complejo como plantean Sotomayor y Dupriez: 
Algunos docentes deben optar por estrategias de más bajo nivel en el plano de la instrucción, otros fortalecerán su rol mediador, otros docentes verán como primera necesidad entregar a sus estudiantes, asistencia social y una acogida efectiva a los niños y niñas, pero sin lugar dudas poder conjugar estos factores equilibradamente aparece como un elemento central de la competencia docente para la enseñanza $(2007$, p. 8$)$.

La falta de adecuación pedagógica re interroga respecto del tipo de formación que se están desarrollando también en otras latitudes. La literatura internacional ha profundizado en esta preocupación (EE-GYEONG, 2011; GALVIS; BONILLA, 2011; INGERSOLL, 1998; JERALD; INGERSOLL, 2002; LU et al., 2007; ZHOU, 2012), llegando a consignar el impacto en el desarrollo social y aprendizaje de los estudiantes. Siendo un elemento transversal los patrones de desigualdad en la distribución de los docentes, reportados también por Clotfelter et al. (2007), Jerald y Ingersoll (2002), Rivero (2015), Schultz (2014) y Zhou (2012). Es decir, se observa la tendencia que articula menor idoneidad disciplinaria a mayor vulnerabilidad del contexto de los estudiantes.

Por otro lado, un desafío clave al que se enfrentan los docentes, es generar aprendizajes asociados a la representación del conocimiento. Ello es más complejo en contextos deprivados socioculturalmente, puesto que muchos de estos estudiantes carecen de hábitos, técnicas y actitudes de estudio. Este escenario se ve aún más difícil, al evidenciar que en Chile la formación inicial docente está centrada en la enseñanza en contextos homogéneos, sin un enfoque estratégico vinculado a la realidad que les tocará enfrentar.

Chile a partir del 2017 ha implementado un nuevo Sistema de Desarrollo Profesional Docente, que según García-Huidobro, necesita de mayor evidencia respecto a los primeros años de docencia en contextos de vulnerabilidad:

La revisión de la investigación en EE.UU. indica que algunas de las estrategias que se prevé usar en Chile podrían ser ineficaces en sectores de pobreza, pues la realidad docente en estos contextos es más compleja que lo que ha aparecido en los debates. En particular, hay una dimensión cultural de la pobreza que afecta directamente a la tarea docente y está casi ausente en la discusión pública (2016, p. 13).

El desempeño docente va más allá de la práctica profesional, requiere un trabajo dinámico en la cual el hacer pedagógico se construye generando una visión sistémica del entorno del estudiante, y donde no basta definir los componentes curriculares, sino que es necesario modelarlos y ejercitarlos en contextos reales y simulados, acompañándolos de procesos de reflexión permanente. Al respecto, Fernández plantea que un gran reto para hacer posible un profesional que ejerza un rol activo en la inclusión educativa es impregnar en los futuros docentes:

Compromiso (voluntad de ayudar a todos lo alumnos), afecto (entusiasmo y cariño hacia los alumnos), conocimiento de la didáctica de la materia enseñada (hacerla accesible para todos), múltiples modelos de enseñanza (flexibilidad y habilidad para resolver lo imprevisto), reflexión sobre la práctica y trabajo en equipo que promueva el aprendizaje entre los colegas, e investigador del propio contexto donde se desenvuelve el alumno (2015, p. 68-69).

En este escenario Calvo, enfatiza, para el análisis desde Latinoamérica, 
[...] la formación de docentes para la inclusión educativa trasciende los programas de formación inicial y amerita programas de formación continua que privilegien el acompañamiento a las prácticas docentes en contextos de vulnerabilidad social. Igualmente requieren procesos de sistematización de experiencias en aras de develar "lecciones aprendidas" que puedan traducirse en recomendaciones para la formación de docentes (2013, p. 29).

Finalmente, mejorar resultados escolares en contextos vulnerables requiere: de prácticas acordes con la diversidad del estudiante y un currículum emergente e integrador con una mirada docente eminentemente inclusiva. Para esto la formación docente inicial y continua es un desafío permanente.

\section{Consideraciones Metodológicas del Estudio}

Se trata de un estudio exploratorio-cualitativo, aplicado a una muestra intencionada de 20 directores de establecimientos escolares particulares subvencionados de la región del Maule (Chile), que han desempeñado el cargo por al menos tres años continuos. Representan el 10\% del total de la subpoblación. Los centros escolares se ubican principalmente en sectores populares, incorporando en su comunidad educativa población estudiantil, con un índice de vulnerabilidad escolar mayor al $50 \%$, lo que representa en enseñanza básica 119 establecimientos (con un 73\% en promedio) y 71 en enseñanza media. Los factores que entregan un grado de homogeneidad importante al ejercicio directivo son: 1) los establecimientos escolares participantes tenían en común que sus propietarios no eran de naturaleza religiosa (más bien laica); 2) tenían una matrícula entre 140 y 260 estudiantes promedio, y un cuerpo docente entre 18 y 33 profesores(as), lo que representa a centros escolares de pequeños a medianos; 3 ) todos los directores tenían una vasta experiencia docente y también directiva, y 17 (de los 20) aún trabajan en aula, práctica habitual en este tipo de establecimientos, que los vincula a una cotidianeidad con sus profesores y su comunidad educativa. Se utilizó una entrevista semiestructurada validada mediante juicio de expertos ${ }^{8}$, por tratarse de un estudio inicial, sus pretensiones son identificar áreas que pudiesen ser investigadas posteriormente; no tiene aspiraciones de representatividad de las temáticas indagadas. En la fase de levantamiento de información, se contactó a cada uno de los directivos previamente seleccionados, explicando el propósito del estudio e invitándoles a participar. Una vez que se obtuvo el consentimiento, se procedió a calendarizar cada entrevista (agosto-noviembre de 2018) en cada establecimiento escolar.

Las entrevistas fueron audio grabadas, siendo posteriormente transcritas para su análisis de contenido. Los resultados fueron ordenados por estamento y unidades de análisis, las cuales fueron generadas a partir de categorías, transcritas y procesadas con el software NVIVO 10.0. La Tabla 1 muestra las dimensiones y categorías que permiten operacionalizar la entrevista.

Una eventual limitación del estudio podría asociarse al hecho que los directivos se refieren al desempeño de otro actor, el profesor. No obstante, creemos que ello es un factor de menor cuantía en este caso, debido a que todos los directivos han tenido una trayectoria importante como docentes de aula en diversos contextos -y muchos de ellos aún la ejercen-, de forma que conocen el escenario, y saben de la situación sobre la cual se les pide su parecer fundado. Adicionalmente, evaluar a sus docentes en algunas dimensiones claves para determinar su continuidad, suele ser un proceder instalado en el sistema desde hace mucho, por lo mismo, esta situación se encuentra razonablemente controlada. 
Tabla 1. Operacionalización de las interrogantes.

\section{Interrogantes utilizadas}

(guion de entrevista)

Directores

Dimensiones

Categoría

¿Qué entiende por vulnerabilidad? y ¿En qué aspectos son vulnerables sus estudiantes?
Vulnerabilidad: concepto y alcances
Contexto Familiar

Riesgo Psicosocial

Capital cultural

Carencias económicas
Competencias conductuales

(Empatía, Compromiso y

¿Cuáles son las características personales y profesionales que poseen los docentes de su establecimiento?
Competencias conductuales

Competencias funcionales
Responsabilidad, Vínculo afectivo)

Competencias funcionales (dominio disciplinario, estrategias didácticas y curriculares, manejo de conflictos)
¿Cómo influyen los docentes en el éxito/fracaso de sus estudiantes bajo esta condición de vulnerabilidad?
Incidencia
Resultados académicos

Desarrollo personal

Fuente: Elaboración propia.

\section{Principales Resultados}

Para los directores, la condición de vulnerabilidad se origina por una combinación de factores, psicosociales, económicos, culturales y del entorno que se conjugan con el estudiante. Más de la mitad de los directores (12 casos, 60\%) menciona que se presenta por el contexto familiar debido a: falta de apego de los padres, familias disfuncionales y monoparentales. En efecto, reconocen que la familia juega un papel relevante, en tanto este conjunto de circunstancias generan un clima poco apto para sobrellevar un proceso educativo eficiente. Urge potenciar o generar herramientas que permitan sobrellevarlos. Una respuesta representativa fue:

Se observan [en los estudiantes] muchas carencias afectivas y emocionales por la familia. Con hogares destruidos que llevan a una baja colaboración de los padres con la educación de sus hijos (entrevistado n. 11).

Nueve casos (45\%) relacionan la vulnerabilidad a riesgo psicosocial del estudiante, que desencadena en condiciones sociales límites y amenazas del entorno, como: precariedad económica, delincuencia y drogadicción. Por ende, señalan que un instrumento esencial en la atención preventiva y de disipación es la escuela.

[...] los estudiantes viven en condiciones precarias que los conduce inevitablemente a muchos a la delincuencia. Muestran serios problemas sociales y de alimentación. Son niños que han sido dañados socialmente. Nuestra escuela está consciente de eso, y lo primero es convencer a las familias de un cambio (entrevistado n. 16).

Finalmente, tres casos (15\%) sitúan la vulnerabilidad como la falta de capital cultural, debido a la baja escolaridad de los padres, analfabetismo y ruralidad, y dos casos (10\%) lo asocian con carencias 
económicas en las familias, producto de bajos ingresos que limitan su calidad de vida. En principio manifiestan que el sistema educativo es parte de la solución, cuando refuerza déficit de conocimientos y de habilidades en sus estudiantes, no obstante, para cambiar la calidad de vida se necesitan acciones -más allá de la escuela- que atiendan situaciones en que una persona siente la falta o privación de algo.

En síntesis, los directores relacionan la vulnerabilidad con una multiplicidad de factores que dificultan el proceso formativo en un estudiante, siendo el principal catalizador su núcleo familiar, reflejando falta de compromiso y de capital cultural, seguido de inconvenientes sociales en donde éste se desarrolla. Potenciar herramientas que aplaquen estos factores de riesgo es tarea ineludible del Estado, que por medio de sus políticas públicas tienen mucho que decir y hacer.

\section{Características de los Docentes de Colegios Vulnerables}

Estas características están ligadas a competencias conductuales y funcionales. Así ocho casos (40\%) declaran que la empatía es la competencia conductual principal del docente para estas realidades. Esto implica tener la capacidad de ponerse en lugar del estudiante, para saber lo que sienten e incluso piensan. Para esto deben estar abiertos al diálogo y a la observación permanente. Algunas respuestas fueron:

[los docentes] deben desarrollar la capacidad de escuchar, comprender y aceptar a sus estudiantes bajo esta condición de difícil educabilidad (entrevistado n. 2).

[los docentes] para mantener la disciplina de los estudiantes primeramente deben saber lo que sienten y piensan ellos (entrevistado n. 10).

Otro grupo de directores (6 casos, 30\%) plantea que los docentes deben desarrollar un alto grado de compromiso y responsabilidad para asumir desafíos que trasciendan de lo pedagógico. Declaran que, junto al compromiso social e institucional, se necesita que asuman la tarea de educar más allá de sus roles habituales. Lo que implica que el docente debe combinar y fortalecer cualidades que superen la simple transferencia de conocimientos.

[los docentes] deben tener una preocupación mayor con los estudiantes y sus familias bajo esta condición (entrevistado n. 16).

[los docentes] deben comprometerse con sus problemas, demostrándoles que tienen la camiseta puesta por ellos (entrevistado n. 13).

Como última competencia conductual (cercana al 15\%), los entrevistados dicen que los docentes deben demostrar un mayor vínculo afectivo/emocional con sus estudiantes. Un buen docente debe establecer conexiones especiales con el estudiantado, demostrando una personalidad alegre y cálida, y una actitud positiva frente a las situaciones problemáticas que se dan en el aula, lo que les permitiría una mayor cercanía.

[los docentes] tienen que tener la capacidad de encantar a sus estudiantes, deben ser mediadores, flexibles y de buen trato (entrevistado n. 20). 
Debe tener un mayor grado de afectividad con los niños y sus familias, sobre todo en momentos difíciles (entrevistado n. 10).

Sobre las competencias funcionales (12 casos, 60\%) indican relevante el dominio disciplinario, entendido como la demostración del conocimiento y manejo de la disciplina que imparten. El docente debe contar con competencias tanto de conocimientos como de experiencia para ejercer su labor. Requisitos elementales para desarrollar un proceso de enseñanza efectivo y sustentable. Algunas respuestas fueron:

[los docentes] deben saber lo que enseñan. Esta es una cuestión vital para el sistema educativo (entrevistado n. 3).

[los docentes] deben demostrar seguridad y dominio de los contenidos de su asignatura (entrevistado n. 6).

En este mismo contexto, seis casos (30\%) aluden al dominio de estrategias didácticas y curriculares, señalando que el docente debe tener la capacidad de seleccionar métodos de enseñanza y recursos educativos pertinentes a las condiciones de sus estudiantes. Implica un conjunto de acciones pedagógicas, que éste debe establecer planificadamente para desarrollar el currículo, intencionado su labor, para la adquisición de aprendizajes significativos en estudiantes que se encuentran en esta situación.

[los docentes] deben gestionar la enseñanza asumiendo el contexto y los recursos pedagógicos con que cuenta la escuela (entrevistado n. 20).

[los docentes] deben ser capaces de organizar y gestionar los contenidos curriculares (entrevistado n. 4).

Aunque en menor recurrencia (menor al 10\%) se planteó que los docentes deben demostrar manejo de conflictos, a través de la capacidad para enfrentar tensiones que se dan en la comunidad educativa. En efecto, deben manejar un conjunto de estrategias que prevengan o confronten hechos problemáticos. El docente debe desarrollar habilidades como: tolerancia, colaboración y confianza para mantener una convivencia pacífica en la escuela.

[los docentes] deben saber manejar los conflictos que se dan a diario acá en la escuela (entrevistado n. 7).

Deben ocupar estrategias para la resolución de problemas disciplinarios con mucha asertividad (entrevistado n. 14).

En resumen, según los directores en las características docentes se deben conjugar competencias conductuales y funcionales, señalando estas últimas como las más relevantes, destacando las relacionadas con el ámbito del conocimiento y la didáctica de la disciplina y el manejo de conflictos. Sin embargo, el abanico de habilidades personales tiende a ser más extenso y en su conjunto poseen mayor peso relativo que el conocimiento técnico, resaltando la empatía, compromiso y la capacidad para crear vínculos afectivos. Condiciones que favorecen un ambiente propicio y apto para el aprendizaje. 
Finalmente, se señala que los docentes resuelvan ciertos desafíos profesionales a la hora de trabajar en establecimientos bajo condiciones de vulnerabilidad: 1) deben contar con perfeccionamiento (herramientas técnicas y sociales) adecuadas, para hacer más efectiva la transmisión del conocimiento y el desarrollo de aspectos emocionales y conductuales en sus estudiantes; 2) lograr en los mismos una formación integral, con aprendizajes significativos, estimulando habilidades emocionales, sociales y éticas, promoviendo un mayor bienestar y calidad en la convivencia social que prevengan conductas de riesgo, como el abandono del sistema escolar.

\section{Influencia Docente en el Desarrollo del Estudiante de Establecimientos Vulnerables}

Más de la mitad de los entrevistados (12 casos, 60\%) señalan que su influencia se observa en el mejoramiento de los resultados académicos. Aluden que el trabajo docente tiene consecuencias concretas en el aprendizaje de los estudiantes, expresado en el aumento en la calificaciones de los exámenes. Entendemos la subjetividad asociada al rendimiento escolar, mirado solo en el cumplimiento de estándares, pero sabemos que el factor docente es el motor principal para conseguirlo. El dinamismo en el proceso de enseñanza que imprime un docente, asociado principalmente a tener altas expectativas, afecta y modifica positivamente el rendimiento del estudiante:

Gracias al desempeño de algunos docentes, los estudiantes han mejorados sus notas considerablemente con respecto al año anterior (entrevistado n. 1).

Las buenas calificaciones son producto de la dedicación del docente, quien cree en sus alumnos y desarrolla sus clases comprometidamente (entrevistado n. 10).

Otro grupo de entrevistados (8 casos, 40\%) menciona que el impacto positivo se presenta en el desarrollo personal del estudiante que se refleja en esta ocasión, en el mejoramiento de la autoestima, la confianza y motivación hacia el estudio. En efecto, estas actitudes y capacidades están relacionadas con procesos de construcción de la identidad personal. Lo que implica que la concepción de calidad educativa debe ser ampliada, pues va más allá del dominio de conocimientos académicos.

Los docentes hacen que los estudiantes se crean el cuento 9 , que sepan que pueden salir de lo que les ha tocado vivir (entrevistado n. 5).

Muchos estudiantes han aumentado su autoestima, que son personas valiosas y que, con esfuerzo y dedicación en el estudio, pueden conseguir todo lo que quieran (entrevistado n. 19).

Seis casos (30\%) reconocen en sus docentes falta de motivación que impacta en su nivel de expectativas. La frustración frente a los bajos resultados académicos, la situación socio afectiva que generan condiciones desfavorables para la enseñanza como: ausentismo escolar, desmotivación, agresividad e inadaptabilidad, evidentemente dificulta el trabajo pedagógico, cuestión que genera en el docente cansancio, observado en un agotamiento mental y físico. Esta situación requiere de mecanismos de atención urgentes, contrariamente las consecuencias para el sistema educativo, pueden ser aún más graves. 
Cuando converso con los docentes manifiestan cansancio físico, desgano y frustración permanente, dado el poco compromiso de los padres, los conflictos y problemas diarios productos de las carencias afectivas de los chicos (entrevistado n. 11).

[los docentes] dicen que bajo estas condiciones les cuesta obtener buenos resultados y que yo no les exija tanto, puesto que se sienten con mucho estrés emocional (entrevistado n. 17).

Finalmente, si bien para algunos directores el bajo rendimiento de los estudiantes es la resultante indirecta de la desmotivación, frustración y bajas expectativas de los docentes, lo cierto es que podría observarse una contradicción pues ellos prefieren mantenerse en la escuela. Algunos directores aluden al clima institucional, entendido como el conjunto de características ambientales que facilitan y estimulan el trabajo.

[los docentes] dicen que en esta escuela hay un buen ambiente laboral y que existe un trato familiar y de respeto (entrevistado n. 10).

[los docentes] aunque existan espacios físicos inapropiados para los aprendizajes, falten materiales de apoyo, igual se quieren quedar acá (entrevistado n. 16).

En síntesis, la influencia del docente se evidencia principalmente en los resultados académicos y el desarrollo personal del estudiante. No obstante, para hacerlo sostenible se necesita atender ciertos aspectos: 1) la labor docente es fundamental, se requiere estar atentos a adecuaciones curriculares innovadoras que involucren el desarrollo, no solo del conocimiento y habilidades, sino también de las actitudes en los estudiantes; 2) aumentar el compromiso de la familia, componente esencial y que en estos ambientes es un indicador bajo. Es más, los directores sienten que las familias traspasan y dejan la responsabilidad en manos del establecimiento; 3 ) fortalecer el ambiente laboral de manera que, frente a la frustración, que en ocasiones sienten los docentes, se activen estrategias de apoyo inmediatas. Los equipos de gestión de los propios establecimientos son los llamados a gestionarlas.

\section{Debate y Propuestas}

En Chile se asume que el director es el gran responsable de los logros o fracasos de su escuela ${ }^{10}$, siendo una amenaza la condición de vulnerabilidad de sus estudiantes, originada en el seno familiar, seguido por el entorno social. Lo cierto es que es una combinación de factores -culturales, sociales y socioeconómico territoriales que llevan inevitablemente a situaciones de riesgo no deseados para el sistema educativo como: ausentismo escolar, indisciplina, desmotivación, expectativas de estudio; que a la larga obstaculiza que los estudiantes alcancen rendimientos académicos y logros relevantes. Es necesario articular un análisis multinivel y un conjunto de procesos inter e intraescuela para combatirlos.

En el plano de las características personales y profesionales que poseen los docentes, los directivos destacan en lo personal la empatía, compromiso y capacidad para crear vínculos afectivos y en lo profesional el conocimiento y didáctica de la disciplina junto al manejo de conflictos. En efecto, este grupo de competencias favorecen la enseñanza, sin embargo y dado el contexto, desarrollar las habilidades personales y sociales en los docentes generan ambientes propicios para el aprendizaje. Lo que requiere poner el acento en mejorar continuamente las prácticas pedagógicas, que involucren el trabajo en equipo, la solución de problemas, la comunicación y manejo del estrés. 
Respecto de la influencia del docente en el desempeño de los estudiantes, los resultados muestran consecuencias positivas, reflejados en el aumento en las calificaciones, y en el desarrollo personal de los mismos. Esto último se traduce en el mejoramiento de la autoestima, confianza y motivación hacia el estudio. Por tanto, las unidades educativas sin abandonar procesos que estimulen el conocimiento y habilidades, deben preocuparse de avanzar en el desarrollo personal y social en sus estudiantes. También los directores reconocen que la influencia del docente en el desempeño de sus estudiantes decrece en ocasiones, por las condiciones desfavorables del ambiente escolar (endógenos y exógenos), cuestión que produce cansancio mental y físico; debilitando su trabajo pedagógico y provocando frustración y baja de expectativas.

Adicionalmente, respecto de las propuestas de apoyo para potenciar la gestión pedagógica en contextos escolares vulnerables, es necesario que se resuelvan ciertos desafíos para trabajar en establecimientos en esta situación:

- Fortalecer las condiciones del trabajo docente de manera que, frente a la desmotivación y bajas expectativas que afectan su compromiso social, se establezcan estrategias de apoyo, gestionadas y articuladas por los equipos de gestión de los establecimientos escolares. Las políticas de mejora venidas desde la perspectiva local tienden a ser más efectivas;

- Contar con docentes con herramientas técnicas y sociales adecuadas para hacer más efectivo el proceso de enseñanza/aprendizaje y el desarrollo de aspectos emocionales y conductuales en sus estudiantes. Esto requiere cambios sustantivos en la formación inicial y continua del docente, aumentando su capacidad de liderazgo de quienes dirigen las escuelas, y una robusta institucionalidad (políticas públicas), como motor de partida para guiar y respaldar los procesos de formación y perfeccionamiento continuo;

- Estimular pedagógicamente en los estudiantes habilidades emocionales, sociales y éticas que prevengan conductas de riesgo de abandono del sistema escolar. Se necesita que los sistemas educativos incentiven el compromiso familiar, con actividades de involucramiento novedosas y apoyos permanentes. Asimismo, establecer métodos y herramientas, basadas (idealmente) en experiencias previas, que experimenten logros iniciales y a escalas en los estudiantes respecto de su falta de compromiso con los estudios, bajas o nulas aspiraciones por continuar su formación, bajo rendimiento académico y actitudes negativas hacia el trabajo escolar. La información de las acciones ejecutadas, permitirá comprobar aciertos para tomar decisiones pertinentes, efectivas y contextualizadas, manteniendo la oportunidad de experimentar, principio esencial para aprender a mejorar;

- En la medida que las prácticas de liderazgo directivo de estos contextos escolares, se centran más en lo técnico pedagógico ( $y$ menos trabajo administrativo), es necesario desplegar mayores conocimientos y potenciar rasgos de carácter en éstos, de manera de afianzar la cohesión, motivación y compromiso con su equipo docente. Esto lleva a implementar comunidades de colaboración en red, capacitadas y empoderadas, de manera de poder compartir herramientas y métodos técnicos pedagógicos efectivos en otras realidades.

Finalmente, para realizar los cambios que se requieren, para alcanzar el éxito de los objetivos en escuelas que concentran población estudiantil altamente vulnerable, se necesitan políticas fuertemente articuladas, con reglas claras para todos, que tiendan a la orientación y desarrollo permanente, independiente del gobierno de turno. 


\section{Contribución de los Autores}

Conceptualización de la investigación, Benavides-Moreno N, Donoso-Díaz S y Reyes-Araya D; Marco teórico, Benavides-Moreno N, Donoso-Díaz S y Reyes-Araya D; Conclusiones, Benavides-Moreno N, Donoso-Díaz S y Reyes-Araya D.

\section{Notas}

1. Ley de Calidad y Equidad de la Educación (LCE) que: 1) fortalece atribuciones del director; 2) configura mecanismos de selección que contribuyan al ejercicio más idóneo de su rol; y 3) le responsabiliza por los resultados que alcanzan los establecimientos educativos.

2. Organismo creado por la Ley n. 20.529. Fiscaliza que los sostenedores de establecimientos educacionales se ajusten a las leyes, reglamentos e instrucciones, exigiéndoles rendiciones de ingresos y gastos, permitiendo la legalidad del uso de los recursos (subvenciones) que el Estado entrega a los establecimientos.

3. Son establecimientos de propiedad y administración privada, pero que, en el caso del nivel parvulario (segundo nivel de transición), enseñanza básica y/o media, reciben financiamiento estatal vía subvención.

4. Organismo de la Administración del Estado, creado en 1964 (Ley n. 15.720), responsable de administrar los recursos estatales de niños, niñas y jóvenes en condición de vulnerabilidad biopsicosocial, para que ingresen, permanezcan y tengan éxito en el sistema educativo.

5. Ministerio de Desarrollo Social de Chile, encargado de promover el desarrollo del país mediante la organización de programas, planes e inversiones públicas.

6. Incorpora variables como: ingreso familiar, ocupación del jefe de hogar, escolaridad de la madre, hacinamiento en el hogar y condición territorial urbano/rural.

7. Sistema Nacional de Asignación con Equidad (SINAE): mide la condición de vulnerabilidad a partir de insumos y características de cada estudiante, que captura la JUNAEB.

8. Se utilizó el Índice de validez de contenido (IVC) de Lawshe (1975).

9. Es un modismo chileno usado para señalar un mejoramiento en la autoestima de una persona, al generar mayor percepción de seguridad en sí misma.

10. Esta situación es más generalizada en el caso de los directores de establecimientos particulares, debido a que poseen atribuciones de contratación y desvinculación directa de sus docentes, salvo excepciones que, por lo general, se encuentran ligadas a establecimientos religiosos. En el caso de la educación pública ello no es así, pues esta atribución es ejercida por el Alcalde o su representante, de forma que los Directores tienen escasa incidencia en esta materia, aunque la ley les faculta para ello.

\section{Referencias}

ARELLANO, J. P. Reforma Educacional. Prioridad que se consolida. Santiago de Chile: Editorial Los Andes, 2000. 
BELLEI, C. ¿Ha tenido impacto la Reforma Educativa Chilena? Santiago de Chile: BID, 2001.

BELLEI, C. El estudio de la segregación socioeconómica y académica de la educación chilena. Estudios Pedagógicos, Valdivia, v. 39, n. 1, p. 325-345, 2013. https:// doi.org/10.4067/S0718-07052013000100019

BELTRÁN, J. Factores que dificultan la gestión pedagógica curricular de los jefes de Unidades Técnico Pedagógicas. Revista mexicana de investigación educativa, Ciudad de México, v. 19, n. 62, p. 939-961, 2014. Disponible en http://www.redalyc.org/pdf/140/14031461014.pdf. Acceso en: 1 ago. 2018.

BRUNNER, J. J.; ELACQUA, G. Factores que inciden en una educación efectiva. Evidencia internacional. \{S. 1.]: Organización de los Estados Americanos, 2006. Disponible en: http://www.opech.cl/bibliografico/ evaluacion/Brunner\%20OEA.pdf. Acceso en: 5 sep. 2015.

CALVO, G. La formación de docentes para la inclusión educativa. Páginas de Educación, Montevideo, v. 6, n. 1, p. 17-33, 2013. Disponible en: http://revistas.ucu.edu.uy/index.php/paginasdeeducacion/issue/ view/22/19. Acceso en: 29 jul. 2018.

CARDEMIL, C. et al. Sistematización proyecto Enseñar para Aprender. Informe interno. CIDE, 2006.

CLOTFELTER, C. et al. High-poverty schools and the distribution of teachers and principals. National Center for Analysis of Longitudinal Data in Education Research, North Carolina, n. 1, p. 1-40, mar. 2007. Working Paper. Disponible en: https://caldercenter.org/sites/default/files/1001057_High_Poverty.pdf. Acceso en: 5 ago. 2018.

COX, C. El esfuerzo de reforma del sistema educativo escolar: Siete Puntos para una Reflexión Docente. Santiago de Chile: MINEDUC, 2001.

DIRECCIÓN GENERAL DE CULTURA Y EDUCACIÓN. Definiciones para la comprensión de situaciones de vulnerabilidad educativa. Dirección Provincial de Planeamiento: La Plata, 2009. p. 2-3. Disponible en: http:// www.region11.edu.ar/publico/portal/doc/biblioteca/vulnerabilidad-educativa.pdf. Acceso en: 15 ene. 2016.

EE-GYEONG, K. Out-of-field secondary school teachers in Korea: their realities and implications. KEDI Journal of Educational Policy, Seúl, v. 8, n. 1, p. 29-48, 2011. Disponible en: https://search.proquest.com/ docview/1013971203? accountid=14675. Acceso en: 10 ago. 2018.

ELACQUA, G.; PACHECO, P. Segregación escolar y políticas públicas. El Mercurio, Santiago de Chile, 9 ene. 2006. Disponible en: http://www.expansiva.cl/columnas/detalle.tpl?idcolumna=01122006102639. Acceso en: 27 jul. 2018.

ESPINOZA, O. et al. Estudiantes vulnerables y sus itinerarios educativos en el sistema escolar municipal en Chile. Revista Iberoamericana de Educación, Madrid, v. 60, n. 4-5, p. 1-16, 2012. https://doi.org/10.35362/ rie6041288

FERNÁNDEZ, J. M. Desafíos de la formación docente ante la exclusión educativa. In: ORTIZ, L. et al. (coords.). Espacios para hablar y compartir sobre la intervención social y educativa en grupos vulnerables. Almería: Enfoques Educativos, 2015. p. 63-70.

FLORES, R. Vulnerabilidad y formación docente, 2009. Trabajo presentado en el seminario El Aprendizaje en Contextos de Vulnerabilidad Escolar, Valparaíso, 2009. 
FULLAN, M.; HARGREAVES, A. La escuela que queremos. Los objetivos por los cuales vale la pena luchar. Buenos Aires: Amorrortu, 1999.

GALVIS, L.; BONILLA, L. Desigualdades regionales en el nivel educativo de los profesores en Colombia. Revista de Economía Institucional, Bogotá, v. 14, n. 26, p. 223-240, 2011. Disponible en: https://www. economiainstitucional.com/pdf/No26/lgalvis.pdf. Acceso en: 10 ago. 2018.

GARCÍA-HUIDOBRO, J. E. La reforma educacional chilena. Madrid: Editorial Popular, 1999.

GARCÍA-HUIDOBRO, J. E. Desigualdad educativa y segmentación del sistema escolar. Consideraciones a partir del caso chileno. Pensamiento Educativo. Revista de Investigación Educacional Latinoamericana, Santiago de Chile, v. 40, n. 1, p. 65-85, 2007. Disponible en: http://pensamientoeducativo.uc.cl/files/journals/2/ articles/399/public/399-897-1-PB.pdf. Acceso en: 26 jul. 2018.

GARCÍA-HUIDOBRO, J. C. Primeros años de docencia en contextos de pobreza: preguntas que la evidencia desde EE.UU sugiere para la conversación en Chile. Pensamiento Educativo. Revista de Investigación Educacional Latinoamericana, Santiago de Chile, v. 53, n. 1, p. 1-16, 2016. https://doi.org/203.262

INGERSOLL, R. The problem of out-of-field teaching. Phi Delta Kappan, Arlington, v. 79, n. 10, p. 773-776, 1998. Disponible en: https://repository.upenn.edu/gse_pubs/137/. Acceso en: 9 ago. 2018.

JERALD, C.; INGERSOLL, R. All talk, no action: Putting an end to out-of-field teaching. The Education Trust, Washington D. C., p. 1-14, ago. 2002. Disponible en: https://repository.upenn.edu/gse_pubs/142/. Acceso en: 9 ago. 2018.

JUNAEB [JUNTA NACIONAL DE AUXILIO ESCOLAR Y BECAS]. Programa de Alimentación Escolar. JUNAEB, 2015. Disponible en https://www.junaeb.cl/programa-de-alimentacion-escolar. Acceso en: 15 ene. 2016.

LAWSHE, C. H. A quantitative approach to content validity. Personnel Psychology, London, v. 28, n. 4, p. 563-575, dic. 1975. https://doi.org/10.1111/j.1744-6570.1975.tb01393.x

LU, X.; SHEN, J.; POPPINK, S. Are teachers highly qualified? A national study of secondary public-school teachers using SASS 1999-2000. Leadership and Policy in Schools, Philadelphia, v. 6, n. 2, p. 129-152, 2007. https://doi.org/10.1080/15700760601168636

MINEDUC [MINISTERIO DE EDUCACIÓN]. Unidad de Gestión Pedagógica y Curricular. Planificación y diseño de la enseñanza. Santiago de Chile: Unidad de Gestión Pedagógica y Curricular, 2008a.

MINEDUC [MINISTERIO DE EDUCACIÓN]. Marco para la buena enseñanza. 7. ed. Santiago de Chile: MINEDUC, 2008b.

RIVERO, M. R. The link of teacher career paths on the distribution of high qualified teachers: a Chilean case study. Educational Policy Analysis Archives, Arizona, v. 23, n. 73, p. 1-35, 2015. https://doi.org/10.14507/ epaa.v23.1710

ROMÁN, M. ¿Por qué los docentes no pueden desarrollar procesos de enseñanza aprendizaje de calidad en contextos sociales vulnerables? Persona y Sociedad, Santiago de Chile, v. 17, n. 1, p. 113-128, 2003. Disponible en: https://dialnet.unirioja.es/ejemplar/73578. Acceso en: 15 ene. 2016. 
ROMÁN, M.; CARDEMIL, C. Sistematización y análisis de los factores asociados al bajo rendimiento escolar en escuelas focalizadas atendidas por el Programa P-900. Informe final. Santiago de Chile: CIDE, 2000.

SCHULTZ, L. Inequitable dispersion: mapping the distribution of highly qualified teachers in St. Louis metropolitan elementary schools. Educational Policy Analysis Archives, Arizona, v. 22, n. 90, p. 1-24, 2014. https://doi.org/10.14507/epaa.v22n90.2014

SOTOMAYOR, C.; DUPRIEZ, V. Desarrollar competencias docentes en la escuela. Aprendizajes de una experiencia chilena de asesoría a escuelas de alta vulnerabilidad social y educativa. Les Cahiers de Recherche en Éducation et Formation, Lovaina, n. 61, p. 389-409, ago. 2007. Disponible en: https://halshs.archivesouvertes.fr/halshs-00561937/document. Acceso en: 09 mar. 2016.

VILLARROEL, D. Competencias profesionales del equipo directivo del sector particular subvencionado chileno en contextos vulnerables. 2014. 301 h. Tesis (Doctorado en Calidad y Procesos de Innovación Educativa) - Facultad de Ciencias de la Educación. Universidad Autónoma de Barcelona, Barcelona, 2014.

WEINSTEIN, J.; MUÑOZ, G. ¿Qué sabemos sobre los directores de escuelas en Chile? Santiago de Chile: Fundación Chile y PUCH, 2012.

ZHOU, Y. Out-of-field teaching: a cross-national study on teacher labor market and teacher quality. 2012. 192 h. Tesis (Doctorado en Filosofía) - Educational Policy. Michigan State University, Michigan, 2012.

\section{Sobre los Autores}

Nibaldo Benavides Moreno es licenciado en Educación por la Universidad de Talca, Chile. Doctor en Educación por la Universidad de Sevilla, España. Líneas de investigación: Análisis de Política y Gestión Educativa; Desarrollo Profesional Docente.

Sebastián Donoso Díaz es licenciado en Educación por la Universidad Católica de Chile. Doctor en Educación por la Universidad Academia de Humanismo Cristiano, Chile. Líneas de investigación: Políticas; Financiamiento; Gestión Educativa Local.

Daniel Reyes Araya es ingeniero Comercial y Magister en Gerencia y Gestión Pública por la Universidad de Talca, Chile. Líneas de investigación: Políticas; Gestión Educativa. 